\title{
Neuroleptoanestesia en Cirugía Pediátrica
}

El uso de un neuroléptico como es el Droperidol, asociado de Fentanyl, analgćsico potente, en proporción de $50 / 1$, permite obtener una neuroleptoanalgesia; si a esta mezcla se le agrega Protóxido de Nitrógeno o Halotano, que permiten la pérdida de la conciencia, se obtiene una neuroleptoanestesia (NLA), técnica anestesiológica que por las excelentes propiedades de sus componentes, tiene indicaciones bien precisas (Tabla No 1).

El Droperidol produce una "neurolepsia", entendiéndose por ésta, un estado de sedación motriz, de indiferencia psíquica y de estabilización netrovegetativa tal, que no impide todos los reflejos útiles y fundamentales del organismo. Con ello se consigue un estado. que no es de steño ni de vigilia, denominado en psiquiatría como de "mineralización".

E1 Droperidol pertenece al grupo de las butirofenoras y se caracteriza por una acción rápida, después de una inyección endovenosa. La acción neuroléptica se manifiesta a los 2-3 minutos, consigutiendo su efecto máximo a los

Tabla Na 1

DROGAS

$\begin{array}{ll}\text { Dehydrobenzperidol (R 4749) } & =\text { neuroléptico } \\ \text { Fentanyl (R 4263) } & \text { analgésico } \\ \text { Thalamonal (R 4749 + R 4263) } & =\text { asociación } \\ & \text { neurolético- } \\ & \text { analgésico } \\ & 50 / 1 .\end{array}$
Río

*Servicio de Anestesiologia, Hospital Roherto del

Rex. Chilem de Pediatria, Vol. 47, No 2, 1976
Dr. Luis Barttlet R.*, Dra. Paulina Riedemann*

10' y su duración es de unos 30 minutos. La duración de la acción total es de 3 a 4 horas. Sus principales propiedades se enumeran en la Tabla No 2.

Tabla N: 2

PROPIEDADES DEL DROPERIDOL

\section{A. Esiabilidad cardiovascular Acción antishock \\ Acción antiarrítmica}

B. Efectos sobre sistena nervioso central.

Potcnte acción anticmética

Sedación psicomotora sin perdida de la conciencia.

Bạa de la presión intraocular

c. Gran margen de seguridad.

No posee efectos toxicos orgánicos

Su acción antiemética es utilísima en el postoperatorio, muy especialmente en niños vomitadores como los pottadores de una piloroestenosis, en los diferentes cuadros intestinales, en cirugía máxilo-facial, en quemados (por razones obvias), (2) en odontología, en otorrinolaringología, en oftalmología, donde además es muy útil la disminución del reflejo óculo cardíaco y de la presión intraocular, especialmente en las operaciones de estrabismo, glaucoma y cataratas. (1)

Su conocido efecto antishock, otra de sus grandes propiedades, por su acción bloqueadora alfa adrenérgica que proporciona una excelente perfusión tisular, (7) es de gran 
ayuda en los niños en shock, ya sea séptico o hipovolémico con la correspondiente reposición en forma adecuada de la volemia en este úlinno, en la cirugía de las malformaciones congénitas del RN. (4) (imperforación ana1, hemia diafragmática, atresias esofágicas $e$ intestinales, quistes lobares congénitos, etc.), en la cirugía de las cardiopatías congénitas, (9) cirugía pulmonar (8) y así en toda la tan variada patología del niño. (5)

Su probada y demostrada acción antiarrítmica es otra contribución valiosa para el trabajo anestésico quirúrgico. (3)

El Fentanyl es un analgésico que pertenece al grupo químico de las 4-acil-anjlino-piperí- dinas y se caracteriza por una acción morfinica típica.

Es el analgésico de acción más rápida, más potente, 100 veces mayor que la morfina y de acción menos duradera conocido hasta ahora.

Después de la inyección endovenosa, su acción analgésica es prácticamente inmediata y su efecto máximo se manifiesta a los 2-3 minutos.

La analgesia quirúrgica útil y suficiente se mantiene durante unos 30 minutos. Con una dosificación adecuada, se consigue una analgesia quirúrgica suficientemente intensa. (6)

Efectos secundarios de estas drogas pueden presentarse, aunque raros; se enumeran cn la Tabla Na 3.

Tabla N.? 3

\section{EFECTOS SECUNDARIOS DE LAS DROGAS USADAS}

\begin{tabular}{|c|c|c|}
\hline Substancias & Electos sectndarios & $\begin{array}{l}\text { Antidotos o substancias } \\
\text { correctoras }\end{array}$ \\
\hline Fentanyl & $\begin{array}{l}\text { Depresión respiratoria } \\
\text { Rigidez torácica } \\
\text { Vagotonia }\end{array}$ & $\begin{array}{l}\text { Nalorfina } \\
\text { Succinilcolina } \\
\text { Atropina }\end{array}$ \\
\hline Dehydrobenzperidol & $\begin{array}{l}\text { Depresión reticular } \\
\text { Depresión neurovegetativa } \\
\text { (acción adrenolítica o } \\
\text { simpaticolítica) } \\
\text { Electos exlrapiramidales }\end{array}$ & $\begin{array}{l}\text { Analépticos } \\
\text { (pretcamida- } \\
\text { anfetaminas) } \\
\text { Simpatícomíméticos } \\
\text { Antiparkinsonianos }\end{array}$ \\
\hline
\end{tabular}

La NLA está indicada en intervenciones que presentan grave riesgo para el paciente, en enfermos cuyo estado general es deficiente o caquéctico, en estados de shock o intoxicados, (8) o que sufren de insuficiencia cardíaca, hepática o renal y que son sometidos a intervenciones graves o de larga duración.

De ahí que a pesar de tener una experiencia numerosa, hemos seleccionado 15 enfermos que por las razones antes expucstas tenían una indicación precisa de esta técnica anestésica,

La edad de los enfermos escogidos en esta casuística y la patologia que presentaban se resumen en las Tablas Nos 4 y 5 .

Tabla N: 4

\section{NEUROLEPTOANESTESIA}

Distribución por edad:

Reciér nacidos y lactantes

Preescolares

0 casos

Escolares

9 casos

6 casos

TOTAL $\overline{15 \text { casos }}$ 
T'abla № 5

\section{NEUROLEPTOANESTESIA}

Diagnósticos:
Fístula intestinal

Lobectomías pulmonares

Broncoscopias

Quistectomías pulm. múltip.

Adenoma suprarrenal

Obstrucción intestinal

Op. de Colonna

Papilomas laríngeas

Amigdalectomía
4 casos

2 casos

2 casos

1 caso

1 caso

1 caso

1 caso

1 caso

1 caso

TOTAL 14 casos

De los 4 casos de fístula intestinal, 3 anestesias corresponden al mismo enfermo, y cl cuarto a un niño con compromiso renal bilateral y sometidos ambos a nutrición parenteral. Por su grave estado y compromiso técnica indicada para ser bien tolerada, ya que la concentración de Fluothano para mantener la pérdida de la conciencia fue mínima.

Los enfermos sometidos a cirugía pulmonar, por el rápido despettar y la analgesia postoperatoria se beneficiaron con la iniciación inmediata del tratamiento kinésico.

En la figura $N^{\circ} 1$ se puede apreciar las de parenquimas vitales, la NLA era la única diferencias de concentración de Fluothano, tanto en la inducción y mantención de la anestesia en los enfermos con y sin NLA. La duración del tiempo operatorio se resume en la Tabla No 6.

Tabla N: 6

NEUROLEPTOANESTESIA

Tiempo operatorio:

\section{DOSIFICACION}

Cada ampolla de Droperidol de $10 \mathrm{cc}$. contiene 25 mgs. y la de FentanyI, también de 10 cc., 0,5 mgr.; se diluyen ambas agregando 15 cc. de suero y se administran según tablas de dosificación especialmente preestablecidas por kg. de peso, ya sea para la inducción y mantención por vía intramuscular o endovenosa stgún se indica en las Tablas $N^{?} 7,8,9$ y 10.

Tabla N? 7

DOSIFICACION DEL DROPERIDOL

Premedicación

Premedicación por vía intramuscular con sol. de Droperidol al $1 \times 1.000$ (to cc. de Droperidol al $2,5 \times 1.000+15 \mathrm{cc}$. de $\mathrm{H}_{2} \mathrm{O}$ destilada) Dosis de $0,20 \mathrm{mg} . \times \mathrm{kg}$. de peso (de sol.

al $1 \times 1.000 \%$.

\begin{tabular}{|c|c|c|c|c|}
\hline \multicolumn{2}{|c|}{ Edad } & \multirow{2}{*}{$\frac{\text { Peso en kgs. }}{3-6}$} & \multirow{2}{*}{$\frac{\text { Dosis en mgs. }}{0,60-1,20}$} & \multirow{2}{*}{$\frac{\text { Volumen en cc. }}{0,6-1,2}$} \\
\hline R. N. & - 6 meses & & & \\
\hline 6 meses & - 1 año & $6-9$ & $1.20-1,80$ & $1.2-1,8$ \\
\hline 1 afio & - 2 años & $9-13$ & $1,80-2,60$ & $1,8-2,6$ \\
\hline 2 años & - 3 аธ̃os & $13-15$ & $2,60-3,00$ & $2,6-3,0$ \\
\hline $\mathfrak{Z}$ años & - 4 años & $15-16$ & $3.00-3.20$ & $3,0-3,2$ \\
\hline 4 años & - 5 ahos & $16-18$ & $3.20-3.60$ & $3.2-3.6$ \\
\hline 5 años & - 6 años & $18-20$ & $3.60-4,00$ & $3.6-4,0$ \\
\hline 6 años & - 7 años & $20-22$ & $4.00-4,40$ & $4,0-4,4$ \\
\hline 7 aก๊ํง & - 8 вйоs & $22-24$ & $4,40-4,80$ & $4.4-4,8$ \\
\hline 8 años & - 9 años & $24-26$ & $4.80-5.20$ & $4,8-5.2$ \\
\hline 9 años & - 10 años & $26-28$ & $5,20-5,60$ & $5.2-5,6$ \\
\hline 10 aก̃os & - 11 años & $28-30$ & $5,60-6,00$ & $5.6-6.0$ \\
\hline I1 años & -12 años & $30-32$ & $6,00-6,40$ & $6,0-6,4$ \\
\hline
\end{tabular}


Con estas dosis los resultados son casi óptimos en cuanto a sedacion psicomotora se refiere, reforzándose además sus otras acciones: antiemética, antishock, antiarrítmica, etc. No hemos observado con esta dosificación efectos colaterales, salvo un caso de un niño de 10 años, grande para nosotros, que presentó una leve crísis oculógira y movimientos fasciculares de los músculos palpebrales que cedieron rápidamente a la inducción anestésica con tiopental.

Tabla N? 8

DOSIFICACION DEL DROPERIDOL

Sal. : $1 \mathrm{mg}, \mathrm{x}$ ce.

Inducción

\begin{tabular}{|c|c|c|c|c|c|c|c|c|c|}
\hline \multicolumn{5}{|c|}{$\begin{array}{l}\text { INTRAMUSCULAR } \\
0,4 \text { mgs. } \times \mathrm{kg} .\end{array}$} & \multicolumn{5}{|c|}{$\begin{array}{l}\text { ENDOVENOSO } \\
0,3 \text { mgs. } x \mathrm{~kg} .\end{array}$} \\
\hline \multicolumn{2}{|c|}{$\begin{array}{c}\text { Peso en } \\
\text { kgs. }\end{array}$} & \multicolumn{2}{|c|}{$\begin{array}{l}\text { Dosis } \\
\text { en mgs. }\end{array}$} & $\begin{array}{l}\text { Volumen } \\
\text { sol. }\end{array}$ & \multicolumn{2}{|c|}{$\begin{array}{l}\text { Peso en } \\
\text { kgs. }\end{array}$} & \multicolumn{2}{|c|}{$\begin{array}{l}\text { Dosis } \\
\text { en mgs. }\end{array}$} & $\begin{array}{l}\text { Volumen } \\
\text { sol }\end{array}$ \\
\hline & kgs. & 1,2 & migs. & $\mathbf{1 , 2} \mathrm{cc}$ & & kgs. & 0,9 & mgs. & 0,9 сc. \\
\hline & $"$ & 1,6 & $"$ & $1,6 "$ & & & 1,2 & $"$ & 1,2 \\
\hline 5 & $"$ & 2,0 & $"$ & $2,0 "$ & 5 & " & 1,5 & " & $1,5 \cdots$ \\
\hline 6 & $"$ & 2,4 & " & $2,4^{\prime \prime}$ & 6 & $"$ & 1,8 & $"$ & $1,8 "$ \\
\hline 7 & $"$ & 2,8 & $"$ & $2,8^{\prime \prime}$ & 7 & $"$ & 2,1 & " & $2,1 "$ \\
\hline 8 & " & 3,2 & $"$ & $3,2^{\prime \prime}$ & 8 & $"$ & 2,4 & $n$ & $2,4^{\prime \prime}$ \\
\hline 9 & $"$ & 3,6 & $n$ & $3,6 \mathrm{n}$ & 9 & $"$ & 2,7 & $"$ & $2,7 \%$ \\
\hline 10 & $"$ & 4,0 & $"$ & $4,0^{\prime \prime}$ & 10 & $"$ & 3,0 & $"$ & $3,0^{\prime \prime}$ \\
\hline 11 & $\eta$ & 4,4 & 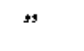 & $4,4^{\prime \prime}$ & 11 & $"$ & 3,3 & " & $3,3 n$ \\
\hline 12 & $"$ & 4,8 & $"$ & $4,8 "$ & 12 & $"$ & 3,6 & " & $3,6 "$ \\
\hline 13 & , & 5,2 & $"$ & $5.2 "$ & 13 & $"$ & 3,9 & " & $3,9 "$ \\
\hline 14 & 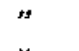 & 5,6 & " & $5,6 "$ & 14 & $"$ & 4,2 & $"$ & $4,2 "$ \\
\hline 15 & 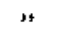 & 6,0 & $"$ & $6,0 "$ & 15 & $"$ & 4,5 & $"$ & $4,5^{\prime \prime}$ \\
\hline 16 & $"$ & 6,4 & $"$ & $6,4 "$ & 16 & $"$ & 4,8 & $"$ & $4,8 "$ \\
\hline 17 & $"$ & 6,8 & $"$ & $6,8 "$ & 17 & $"$ & 5,1 & $"$ & $5,1 "$ \\
\hline 18 & " & 7,2 & $"$ & $7,2 "$ & 18 & $"$ & 5,4 & $"$ & $5,4^{n}$ \\
\hline 19 & $"$ & 7,6 & $"$ & $7,6^{\prime \prime}$ & 19 & " & 5,7 & $"$ & $5,7 "$ \\
\hline 20 & $"$ & 8,0 & $"$ & $8,0 "$ & 20 & $"$ & 6,0 & $"$ & $6,0^{\prime \prime}$ \\
\hline 21 & " & 8,4 & " & $8,4^{\prime \prime}$ & 21 & $"$ & 6,3 & " & $6,3 "$ \\
\hline 22 & $"$ & 8,8 & $"$ & $8,8 "$ & 22 & $"$ & 6,6 & $n$ & $6,6 "$ \\
\hline 23 & $"$ & 9,2 & $"$ & $9,2 "$ & 23 & $"$ & 6,9 & $"$ & $6,9^{\prime \prime}$ \\
\hline 24 & " & 9,6 & $"$ & $9,6 "$ & 24 & $"$ & 7.2 & $"$ & $7,2 "$ \\
\hline $2 \bar{j}$ & * & 10,0 & $"$ & $10,0^{\prime \prime}$ & 25 & $"$ & 7,5 & $"$ & $7,5 "$ \\
\hline 26 & $"$ & 10,4 & " & $10,4 "$ & 25 & "' & 7,8 & " & $7,8 "$ \\
\hline 27 & $s$ & 10,8 & $"$ & 10,8 & 27 & " & 8,1 & " & $8,1^{\prime \prime}$ \\
\hline 28 & " & 11,2 & $"$ & $11,2 "$ & 28 & $"$ & 8,4 & $"$ & $8.4^{\prime \prime}$ \\
\hline 29 & $"$ & 11,6 & $"$ & $11,6 "$ & 29 & $"$ & 8,7 & $"$ & $8,7 "$ \\
\hline 50 & " & 12,0 & " & $12,0^{\prime \prime}$ & 30 & $"$ & 9,0 & $"$ & $9,0 "$ \\
\hline 31 & $"$ & 12,4 & $"$ & $12,4 "$ & 31 & $"$ & 9,3 & $"$ & 9,3 \\
\hline 32 & $"$ & 12,8 & " & $12,8 "$ & 32 & $"$ & 9,6 & $"$ & $9,6^{\prime \prime}$ \\
\hline
\end{tabular}


DOSIFICACION DEL FENTANYL

10 cc. Fentanyl +15 cc. SF. $(0,02 \mathrm{mg} \cdot \mathrm{x}$ cc. $)$

PREMEDICACION

$0,003 \mathrm{mg} . \times \mathrm{kg}$. peso

0,15 cc. solución
INDUCCION Intramuscular

$0,010 \mathrm{mg}, x \mathrm{~kg}$.

0,5 cc. solución

\begin{tabular}{|c|c|c|c|c|c|c|c|c|}
\hline \multicolumn{2}{|c|}{$\begin{array}{c}\text { Peso en } \\
\mathrm{kgs} .\end{array}$} & \multicolumn{2}{|c|}{$\begin{array}{c}\text { Dosis en } \\
\text { mgs. }\end{array}$} & \multirow{2}{*}{$\begin{array}{c}\begin{array}{c}\text { Volumen } \\
\text { eft cc. }\end{array} \\
0,45 \mathrm{cc} .\end{array}$} & $\begin{array}{c}\text { Pesso et } \\
\quad \mathrm{kgs.}\end{array}$ & \multicolumn{2}{|c|}{$\begin{array}{c}\text { Dosis ent } \\
\text { mgs. }\end{array}$} & $\begin{array}{l}\text { Volumen de } \\
\text { sol. en ce. }\end{array}$ \\
\hline & kgs. & 0,009 & mgs. & & $3 \mathrm{kgs}$. & 0,030 & mgs. & $1,5 \mathrm{cc}$. \\
\hline 4 & " & 0,012 & $"$ & $0,60 "$ & $4 "$ & 0,040 & $"$ & $2.0 "$ \\
\hline $\bar{j}$ & . & 0,015 & $"$ & $0,75^{\prime \prime}$ & $5 "$ & 0,050 & $"$ & $2,5^{\prime \prime}$ \\
\hline 6 & $"$ & 0,018 & " & $0,90 "$ & $6 \%$ & 0,060 & " & $3,0 "$ \\
\hline 7 & $"$ & 0,021 & $"$ & $1,05^{\prime \prime}$ & $7 "$ & 0,070 & ני & 3,50 \\
\hline 8 & $"$ & 0,024 & $"$ & $1,20 "$ & 8 " & 0,080 & $"$ & $4,0^{\circ \prime}$ \\
\hline 9 & $"$ & 0,027 & $"$ & $1,35 "$ & $9 "$ & 0,090 & $n$ & $4,5^{\prime \prime}$ \\
\hline 10 & $"$ & 0,030 & $"$ & $1,50 "$ & $10 "$ & 0,10 & $n$ & $5,0 "$ \\
\hline 11 & $"$ & 0,033 & , & $1,65 "$ & $11 "$ & 0,11 & " & $5,5 "$ \\
\hline 12 & $"$ & 0,036 & $"$ & $1,80 "$ & $12 "$ & 0,12 & $"$ & $6,0^{\circ}$ \\
\hline 13 & $"$ & 0,039 & " & $1,95 "$ & $13 "$ & 0,13 & $"$ & $6,5 "$ \\
\hline 14 & $"$ & 0,042 & $"$ & $2,10 "$ & $14 "$ & 0,14 & $"$ & $7,0 "$ \\
\hline 15 & $"$ & 0,045 & $"$ & $2,25 "$ & $15 "$ & 0,15 & " & $7,5 "$ \\
\hline 16 & $"$ & 0,048 & $"$ & $2,40^{\circ}$ & $16 "$ & 0,16 & $"$ & $8,0 "$ \\
\hline 17 & $"$ & 0,051 & " & 2,55 & $17 "$ & 0,17 & $"$ & $8,5 "$ \\
\hline 18 & $"$ & 0,054 & " & $2,70^{\prime \prime}$ & $18 "$ & 0,18 & $"$ & $9,0 "$ \\
\hline 19 & $"$ & 0,057 & $"$ & $2,85 "$ & $19 "$ & 0,19 & $"$ & $9,5 "$ \\
\hline 20 & $"$ & 0,050 & $"$ & $3,00 "$ & $20 "$ & 0,20 & $"$ & $10,0 "$ \\
\hline $2 \mathrm{I}$ & $n$ & 0,063 & $"$ & $3,15 "$ & $21 "$ & 0,21 & $"$ & $10,5 "$ \\
\hline 22 & $"$ & 0,066 & $n$ & $3,30 \mathrm{k}$ & $22 "$ & 0,22 & $\Rightarrow$ & $11,0 "$ \\
\hline 23 & $"$ & 0,069 & $"$ & $3,45 "$ & $23 "$ & 0,23 & $"$ & $11,5 "$ \\
\hline 24 & $"$ & 0,072 & $"$ & $3,60 "$ & $24 "$ & 0,24 & $"$ & $12,0 "$ \\
\hline 25 & $"$ & 0,075 & $"$ & $3,75 "$ & $25 "$ & 0,25 & $"$ & $12,5 "$ \\
\hline 26 & $"$ & 0,078 & $"$ & $3,90 "$ & $26 "$ & 0,26 & $"$ & $13,0 "$ \\
\hline 27 & $"$ & 0,081 & $"$ & $4,05 "$ & $27 "$ & 0,27 & $"$ & $13,5 ”$ \\
\hline 28 & $"$ & 0,084 & $"$ & $4,20 "$ & $28 "$ & 0,28 & $"$ & $14,0 "$ \\
\hline 29 & $"$ & 0,087 & $"$ & $4,35 "$ & $29 "$ & 0,29 & $"$ & $14,5 "$ \\
\hline 30 & $"$ & 0,090 & $"$ & $4,50 "$ & $30^{\prime \prime}$ & 0,30 & $"$ & $15,0 "$ \\
\hline 31 & $"$ & 0,093 & $"$ & $4,65 "$ & $31 "$ & 0,31 & $"$ & $15,5 "$ \\
\hline 32 & $"$ & 0,096 & $"$ & $4,80 "$ & $32 "$ & 0,32 & $"$ & $16,0^{\prime \prime}$ \\
\hline
\end{tabular}


DOSIFICACION DEL FENTANYL

Sol. 0,02 mg. $x$ ce.

Mantención

\begin{tabular}{|c|c|c|c|c|c|c|c|c|c|c|c|}
\hline \multicolumn{5}{|c|}{$\begin{array}{l}0,0015 \text { mgs. } \mathrm{x} \text { kg. } \\
\text { Endovenoso }\end{array}$} & \multicolumn{3}{|c|}{$\begin{array}{l}0,0020 \text { mgs. } \mathrm{x} \mathrm{kg} . \\
\text { Endoyenoso }\end{array}$} & \multicolumn{4}{|c|}{$\begin{array}{c}\text { Jntramuscular } \\
0,0050 \text { mgs. } x \mathrm{~kg} \text {. }\end{array}$} \\
\hline \multicolumn{2}{|c|}{$\begin{array}{l}\text { Peso } \\
\text { (kgs.) }\end{array}$} & \multicolumn{2}{|c|}{$\begin{array}{l}\text { Dosis } \\
\text { (mgs.) }\end{array}$} & $\begin{array}{c}\text { Volumen de } \\
\text { sol. (cc.) }\end{array}$ & \multicolumn{2}{|c|}{$\begin{array}{l}\text { Dosis } \\
\text { (mgs.) }\end{array}$} & $\begin{array}{c}\text { Voltumen de } \\
\text { sol. (cc.) }\end{array}$ & \multicolumn{2}{|c|}{$\begin{array}{l}\text { Dosis } \\
\text { (mgs. J }\end{array}$} & \multicolumn{2}{|c|}{$\begin{array}{l}\text { Volumen de } \\
\text { sol. (cc.) }\end{array}$} \\
\hline & kgs. & $0,0045 \mathrm{n}$ & mgs. & $0,225 \mathrm{cc}$ & $0,0060 \mathrm{r}$ & mgs. & $0,3 \mathrm{cc}$ & 0,009 & mgs. & 0,45 & cc. \\
\hline 4 & $"$ & 0,0060 & $"$ & $0,300 "$ & 0,0080 & $"$ & $0,4 "$ & 0,012 & $"$ & 0,60 & $"$ \\
\hline 5 & $"$ & 0,0075 & $"$ & $0,375 "$ & 0,010 & $"$ & $0,5 "$ & 0,015 & $"$ & 0,75 & $"$ \\
\hline 6 & $"$ & 0,0090 & $"$ & $0,450 "$ & 0,012 & $"$ & $0,6 "$ & 0,018 & $"$ & 0,90 & $"$ \\
\hline 7 & $"$ & 0,0105 & $"$ & $0,525 "$ & 0,014 & $"$ & $0,7 "$ & 0,021 & $"$ & 1,05 & $"$ \\
\hline 8 & $"$ & 0,0120 & $"$ & $0,600 "$ & 0,016 & $"$ & $0,8 *$ & 0,024 & $"$ & 1,20 & $"$ \\
\hline 9 & $"$ & 0,0135 & $"$ & $0,675 "$ & 0,018 & $"$ & $0,9 "$ & 0,027 & $"$ & 1,35 & $"$ \\
\hline 10 & $"$ & 0,0150 & $"$ & $0,750^{\prime \prime}$ & 0,020 & 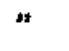 & $1,0 "$ & 0,030 & $"$ & 1,50 & $"$ \\
\hline 11 & $"$ & 0,0165 & $"$ & 0,825 & 0,022 & $"$ & $1,1 "$ & 0,033 & $"$ & 1,65 & $"$ \\
\hline 12 & $"$ & 0,0180 & $"$ & $0,900 "$ & 0,024 & $"$ & $1,2 "$ & 0,036 & $"$ & 1,80 & $"$ \\
\hline 13 & $"$ & 0,0195 & $"$ & $0,975 "$ & 0,026 & $"$ & $1,3 "$ & 0,039 & $"$ & 1,95 & $"$ \\
\hline 14 & $"$ & 0,0210 & $"$ & $1,050 "$ & 0,028 & $"$ & $1,4 "$ & 0,042 & $"$ & 2,10 & $"$ \\
\hline 15 & $"$ & 0,0225 & $"$ & $1,125 "$ & 0,030 & $"$ & 1,5" & 0,045 & $"$ & 2,25 & $"$ \\
\hline 16 & $"$ & 0,0240 & $"$ & $1,200^{* \prime}$ & 0,032 & $"$ & $1,6 "$ & 0,048 & $"$ & 2,40 & $"$ \\
\hline 17 & $"$ & 0,0255 & $*$ & $1,275 "$ & 0,034 & $"$ & $1,7 "$ & 0,051 & $"$ & 2,55 & $"$ \\
\hline 18 & $"$ & 0,0270 & $"$ & $1,350 "$ & 0,036 & $"$ & $1,8 "$ & 0,054 & $"$ & 2,70 & $"$ \\
\hline 19 & $"$ & 0,0285 & $"$ & $1,425 "$ & 0,038 & $"$ & $1,9 "$ & 0,057 & $"$ & 2,85 & $"$ \\
\hline 20 & $"$ & 0,0500 & $"$ & $1,500^{n}$ & 0,040 & $"$ & $2,0 "$ & 0,060 & $"$ & 3,00 & $"$ \\
\hline 21 & $"$ & 0,0315 & $"$ & $1,575 "$ & 0,042 & $"$ & $2,1 "$ & 0,063 & $"$ & 3,15 & $"$ \\
\hline 22 & $"$ & 0,0330 & $"$ & $1,650 "$ & 0,044 & $"$ & $2,2 "$ & 0,066 & $"$ & 3,30 & $"$ \\
\hline 23 & $"$ & 0,0345 & $"$ & $1,725^{\prime \prime}$ & 0,046 & $"$ & $2,3 "$ & 0,069 & $"$ & 3,45 & $"$ \\
\hline 24 & $"$ & 0,0360 & $"$ & $1,800 "$ & 0,048 & $"$ & $2,4 "$ & 0,072 & $n$ & 3,60 & $"$ \\
\hline 25 & $"$ & 0,0375 & $"$ & $1,875 "$ & 0,050 & $"$ & $2,5 \mathrm{M}$ & 0,075 & $"$ & 3,75 & $"$ \\
\hline 26 & $"$ & 0,0390 & $"$ & $1,950 "$ & 0,052 & נ & $2,6 "$ & 0,078 & $"$ & 3,90 & $"$ \\
\hline 27 & $"$ & 0,0405 & $"$ & $2,025 "$ & 0,054 & $"$ & $2,7 "$ & 0,081 & $"$ & 4,05 & $"$ \\
\hline 28 & $n$ & 0,0420 & $"$ & $2,100 "$ & 0,056 & $n$ & $2,8 "$ & 0,084 & $"$ & 4,20 & $"$ \\
\hline 29 & $"$ & 0,0435 & $"$ & $2,175^{\prime \prime}$ & 0,058 & $"$ & $2,9 "$ & 0,087 & $"$ & 4,35 & $"$ \\
\hline 30 & $"$ & 0,0450 & $"$ & $2,250 "$ & 0,060 & $"$ & $3,0 \%$ & 0,090 & $"$ & 4,50 & $"$ \\
\hline 31 & $"$ & 0,0465 & $"$ & $2,325 "$ & 0,062 & $"$ & $3,1 "$ & 0,093 & $"$ & 4,65 & $"$ \\
\hline 32 & $"$ & 0,0480 & $"$ & $2,400 "$ & 0,064 & $"$ & $3,2 "$ & 0,096 & $"$ & 4,80 & $"$ \\
\hline
\end{tabular}



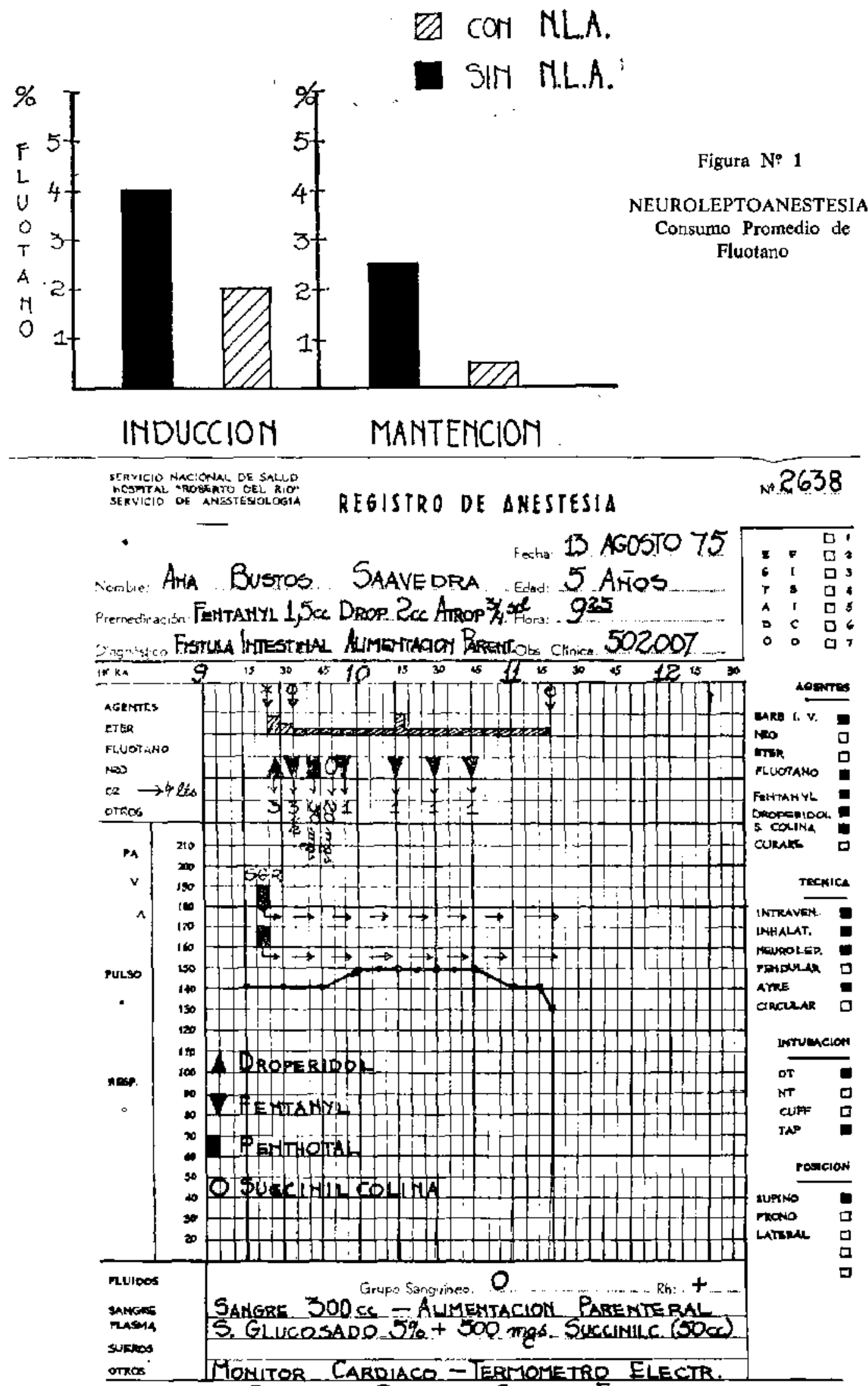

OTRO CO HOMITOR CARDIACO - ERMOMETRO ELECTR

Operocion ciectráa REseccion PARCLA $\rightarrow$ CIERRE FISTULAS 
La téenica auestésica se resume en el Registro de Anestesia de uno de los pacientes (Figura No 2).

\section{RESUMEN Y COMENTARIO}

Hemos querido presentar nuestra experiencia con Neuroleptoanestesia, comprobando que se trata de una técnica anestesiológica que tiene indicaciones bien precisas, especialmente en enfermos de gran riesgo quirúrgico y que nos proporciona una mínima toxicidad general, gran margen de seguridad, estabilidad cardiovascular, protección efectiy: contra los vómitos y shock traumático, restablecimiento postoperatorio rápido y favorable. Recslcamos que se trata de un procedimiento anestésico que se usa desde hace muchos años, pero que en .Cirugía Pediátrica, al menos en nuestro medio ha sido escasamente divulgado.

\section{SUMMARY}

We presented our experience with NLA in infants and children. When good elected it is a very good tecnic, specially in high risk patients. It has low toxicity, great security and cardiac balance. Its antiemetic and antishock effects give a good post operatjye period. It is not a new precedure, but it has been used rarely in childhbod in our country.

\section{REFERENCIAS}

1. Ivankovic, A. D. and H. I. Lowe: Anesth. and Analg. 48: 933-938, 1969. Nov.

2. Baskett, P. J. F.: Postgrad. Med. J. 48: 138-142, 1972.

3. Goldberg, A. H. and Ch. Padget: Anesth. and Analg. 48: 978.982, 1969. Nov.-Dec.

4. Moran, I. H. and B. M. Marshall: Can. Anest. Soc. J. 13: 272, 1966.

5. Aubuy, V. et al.: Can. Anest. Soc. I. 13: 263, 1966.

6. Cabrera, G, L. ef al.: Arch. Soc. Cir. Chile. 1964. Mayo.

7. Gemperle, $M$. and $B$, Grilnniger: Anesthesist. 13: 6, 1964.

8. Ceraso, $O$. et al.: 24: Congreso Argentino de Citugía. Oct. 7-11, 1963.

9. Corssen, $G$. et al.; J. Thoracic and Candiovasc. Sur. 49: 904, 1965. 